\title{
Insegnare la storia del lavoro. L'esperienza di Fondazione Isec
}

\author{
di Monica Di Barbora
}

Mag 12, 2020 | In evidenza, Insegnare la contemporaneità oggi | $\underline{0} \mid$

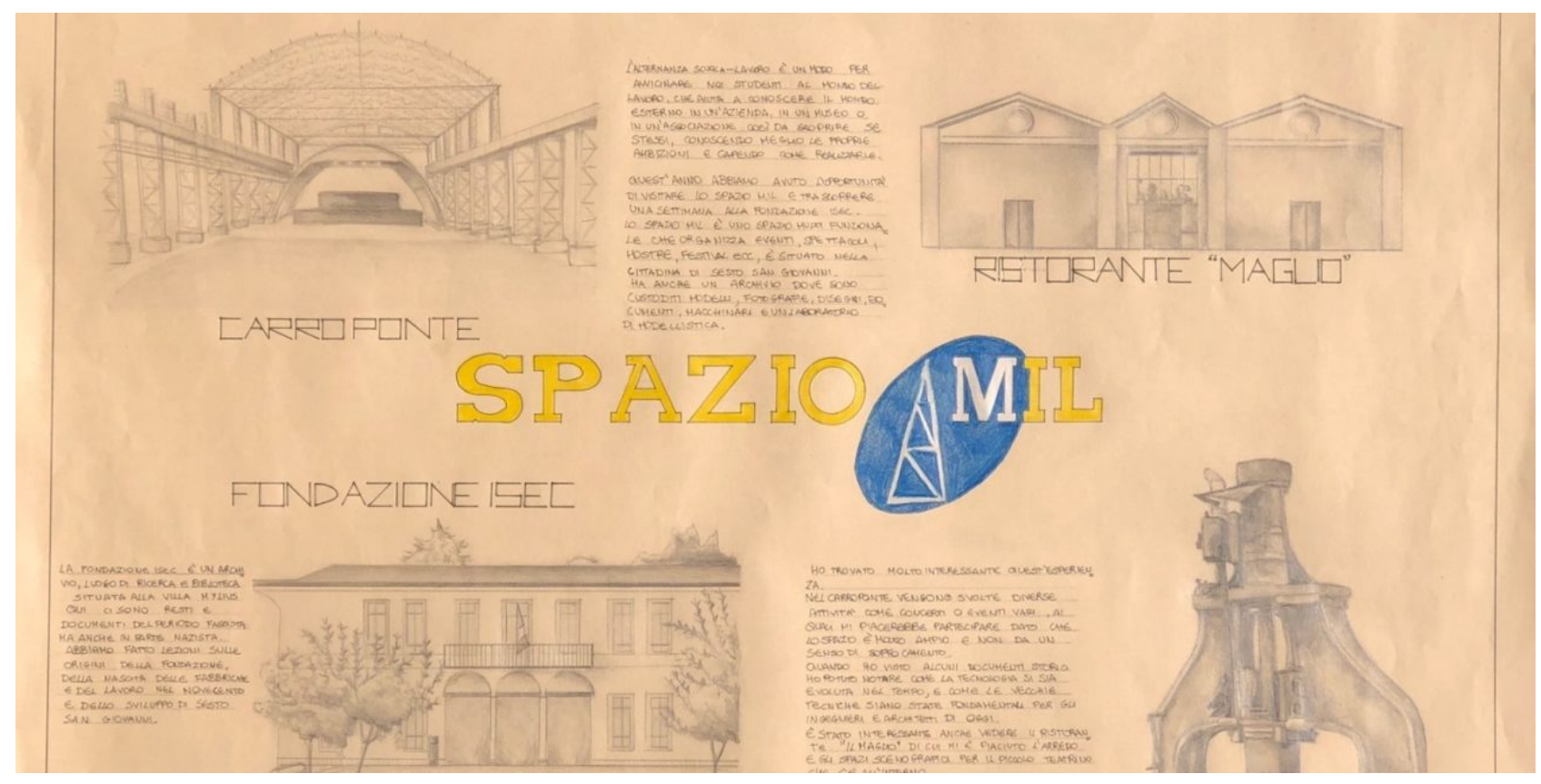

Un pannello della mostra conclusiva (Rosa Ramondo, 2019)

\begin{abstract}
L'articolo riflette sul senso dell'inserimento della storia del lavoro nei percorsi scolastici e su alcune modalità attraverso cui essa può essere proficuamente insegnata. Lo fa attraverso la presentazione di due esperienze organizzate dalla Fondazione Isec di Sesto San Giovanni. L'autrice individua alcuni elementi che possono rendere affascinante per gli adolescenti un argomento complesso da trasmettere e spesso lontano dai loro interessi. Uno degli elementi che vengono indicati è la pluralità di strategie e professionalità coinvolte nel processo. Un ulteriore suggerimento è quello di partire dalla materialità dei luoghi e degli oggetti. Infine, si conferma l'utilità di coinvolgere le classi in attività da compiere in prima persona (laboratori sui documenti di archivio, interviste a testimoni, prodotti di restituzione finale).
\end{abstract}

\section{Scuola e mondo del lavoro}

Il collegamento tra scuola e mondo del lavoro è uno tra gli aspetti più delicati e discussi della riflessione sul nostro sistema educativo, non solo tra le professionalità direttamente interessate al tema ma anche nel dibattito pubblico. Se molti attacchi al sistema scolastico partono proprio dall'accusa di non saper costruire, nelle alunne e negli alunni, una strutturata professionalità che sia in diretta continuità con il mondo del lavoro, altre posizioni sottolineano invece il ruolo della scuola esclusivamente come agente promotore di cultura, come spazio in cui costruire delle menti aperte e delle persone capaci di riflessione critica e autonomia di pensiero e considerano questo come il miglior viatico per il mondo, anche professionale, con cui le/i giovani dovranno confrontarsi. Su 
questi temi il Ministero sembra dare risposte ondivaghe: dopo avere inserito bruscamente un altissimo monte ore dedicato all'Alternanza scuola lavoro, queste sono state dimezzate, assumendo inoltre la denominazione di Percorsi per le competenze trasversali e l'orientamento (Pcto), in cui è evidente il tentativo di riportare questo tipo di attività a forme di costruzione delle competenze di cittadinanza, con la scomparsa del termine «lavoro» dal titolo.[1] Sostantivo che torna, invece, nella Legge 92 del 2019 (si tratta della norma che reintroduce l'insegnamento dell'educazione civica) ma esclusivamente in relazione al diritto (tra le tematiche da privilegiare si inseriscono, all'articolo 3, «elementi fondamentali di diritto, con particolare riguardo al diritto del lavoro»). La situazione è, insomma, fluida e necessiterebbe di una riflessione più approfondita e di una seria discussione più ampiamente condivisa con insegnanti e esperti del settore.[2]

Creare una scuola che abbia come priorità esclusivamente l'accesso al lavoro, quasi a considerare una perdita di tempo lo studio e l'approfondimento culturale fini a se stessi, rischia di rivelarsi, sulla media e lunga distanza, controproducente se non dannoso per i singoli e per la società nel suo insieme. Tuttavia è evidente che lo spazio riservato all'economia e ai meccanismi del sistema produttivo, che pure condizionano profondamente la vita di ognuno di noi, è praticamente inesistente a scuola. Una declinazione del binomio scuola/lavoro incardinata sulla trasmissione di alcuni elementi di storia del lavoro, anche in relazione all'espansione e alla contrazione dei diritti correlati, potrebbe allora essere un arricchimento importante. Aiutare la riflessione su questi temi proponendo percorsi sulla storia del lavoro e sul modo in cui la produzione modifica le relazioni sociali e il territorio in cui viviamo - è un modo per irrobustire significativamente gli strumenti di analisi della realtà contemporanea a disposizione delle giovani e dei giovani. Per far crescere quella capacità di riflettere criticamente sul passato e sul presente che dovrebbe essere compito principale di un buon sistema educativo.

\section{La didattica in contesto}

Fondazione Isec è nata in un contesto pesantemente condizionato proprio dalla massiccia presenza di alcune grandi aziende, tra le più importanti del Paese: Breda, Marelli, Falck, Campari, Pirelli, per richiamare solo alcuni esempi. All'inizio del XX secolo, gli abitanti di Sesto San Giovanni erano 6952; dieci anni dopo, con la comparsa delle prime fabbriche, il numero era già praticamente raddoppiato e i cittadini raggiungevano le 13667 unità. Nel 1963 la città sarebbe arrivata a 70.000 abitanti con una estensione di 3 milioni di metri quadri di superficie produttiva: il piccolo borgo agricolo si era trasformato in una delle aree industriali tra le più grandi d'Europa. 


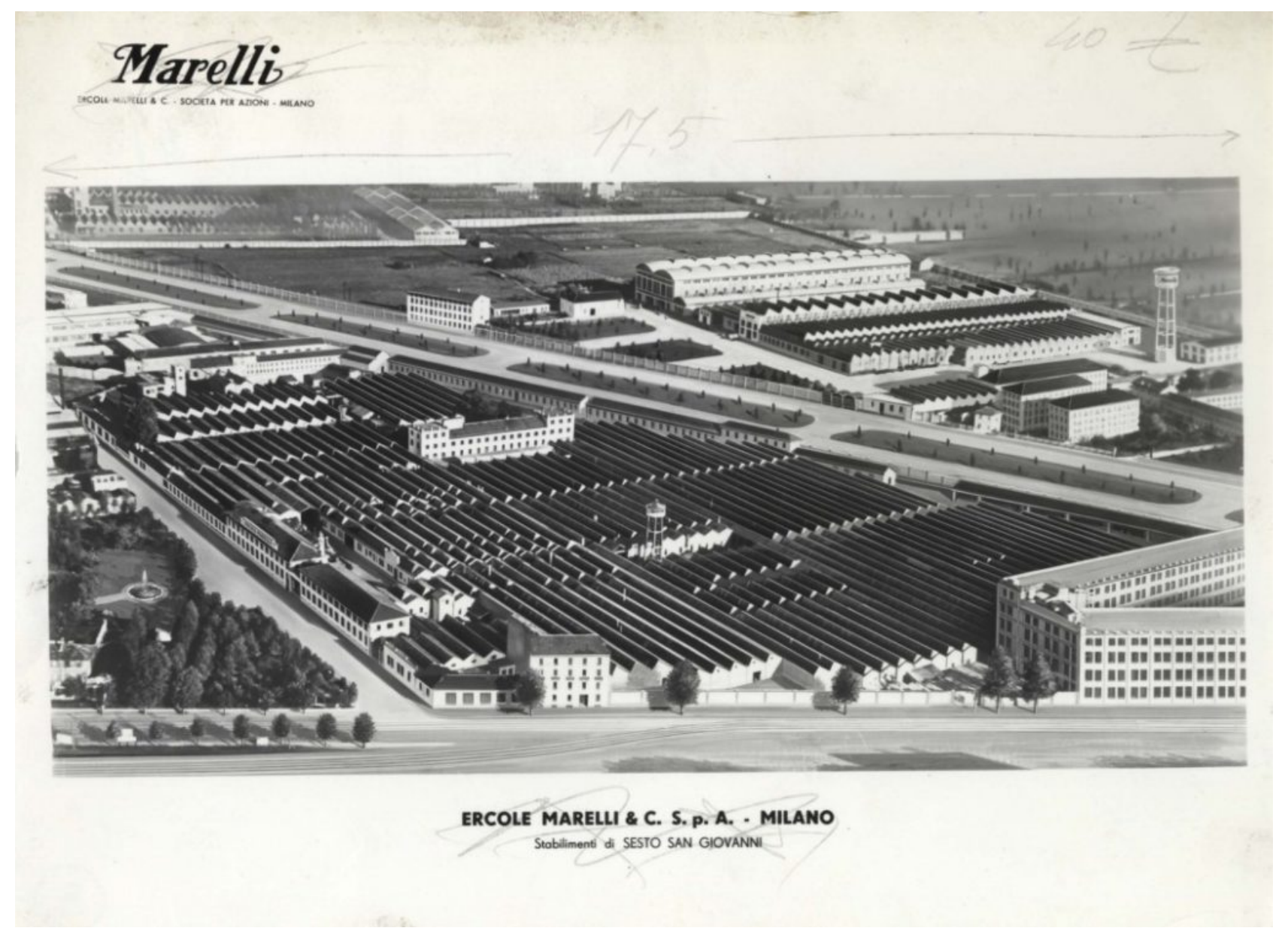

Società italiana Ernesto Breda per costruzioni meccaniche, sezione siderurgica, laminatoio da 600 mm, Sesto San Giovanni, circa 1920 (Fondazione ISEC, Sezione fotografica dell'Archivio storico Breda, AL30_008) 


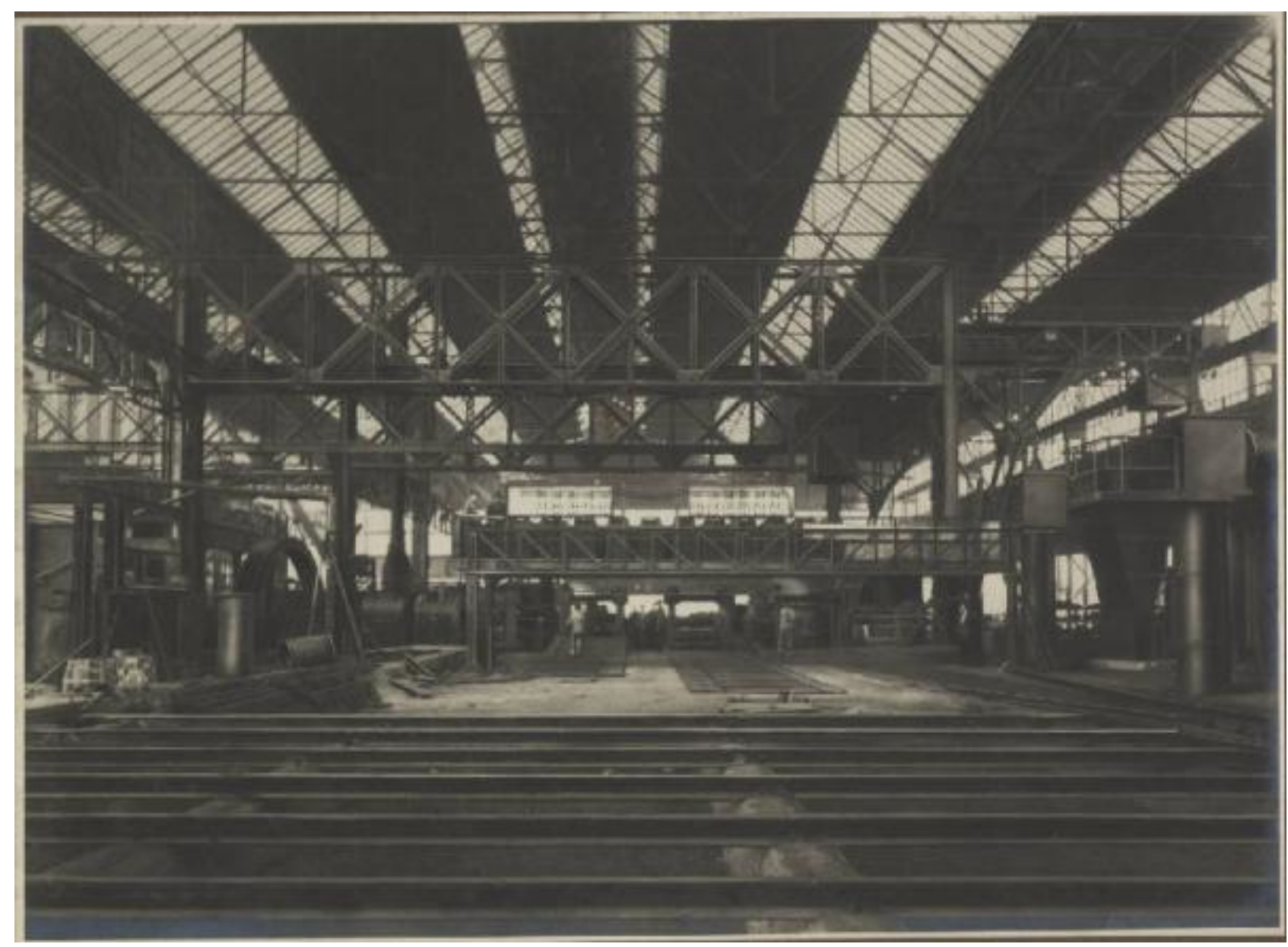

La Magneti Marelli a Sesto San Giovanni, 1953

La Fondazione nasce, nel 1973, proprio per riflettere su questo impressionante mutamento, per studiare la vita nelle fabbriche e per raccogliere la documentazione che si era andata via via stratificando. Nel corso degli anni, il patrimonio archivistico raggiunge così i due chilometri lineari a cui si aggiunge una biblioteca specializzata sulla storia sociale della contemporaneità, in particolare proprio la storia del lavoro.

Le proposte didattiche su questo tema si incardinano, quindi, su un particolare contesto territoriale e sulla specificità dell'istituzione che le struttura.

\section{Due progetti speciali}

Il tema della storia del lavoro è, quindi, connaturato alla nascita e allo sviluppo stessi della Fondazione ed è costantemente presente, con varie declinazioni, nell'offerta formativa e didattica annuale. Due progetti, in particolare, consentono, per estensione e articolazione, di mostrare le risorse e le opportunità attivate da questi percorsi.

Si tratta di un seminario di due giorni proposto a una classe del liceo cantonale a indirizzo economico di Mendrisio e di una collaborazione ormai pluriennale con un istituto superiore milanese, il Liceo artistico statale "Brera", su progetti di Alternanza scuola lavoro (e ora di Percorsi per le competenze trasversali e l'orientamento).

\section{Storia del lavoro e della fabbrica nel Novecento}


Il primo progetto, strutturato su due giornate intere, compone un mosaico di attività didattiche differenti attuate da interlocutori diversi: lezioni frontali, laboratori in archivio, visite guidate, incontri con i testimoni e intervalli conviviali in cui gli studenti possono confrontarsi tra loro e con alcuni dei docenti sui temi trattati. Una strategia didattica semplice ma efficace per mantenere desta l'attenzione e, contemporaneamente, rappresentare e proporre punti di vista molteplici.

Dopo l'accoglienza iniziale, alla classe viene presentata, in una lezione frontale, la vicenda dell'industrializzazione sestese, a partire dallo spazio stesso in cui ci si trova. Villa Mylius, infatti, sede della Fondazione in cui si tengono laboratori e lezioni, è stata costruita da un industriale tedesco del settore tessile che, oltre alla residenza principale nel centro di Milano, fece costruire, o riadattare (gli archivi purtroppo non sono chiari su questo punto), questo edificio in un'area allora agricola. La struttura abbinava le classiche caratteristiche di una villa di delizia al fine pratico dell'allevamento dei bachi da seta e della sperimentazione della coltivazione di piante diverse all'interno della serra e del parco.

Le vicende, narrate a partire dagli spazi stessi in cui hanno avuto luogo, perdono la lontananza indistinta e quasi favolosa in cui sembra che cadano tutti gli eventi del passato nella percezione degli studenti, per prendere corpo in elementi architettonici e naturali (la parte sopravvissuta del giardino).

Questa prima parte della narrazione dello sviluppo storico viene completata, poi, dalla passeggiata alla scoperta delle aree di archeologia industriale con una guida specializzata. Nuovamente, la narrazione delle vicende storiche si inserisce nello spazio che esse hanno contribuito a creare. Gli spazi industriali, le fabbriche, o quello che ne rimane, circondano gli studenti che sono stimolati, anche visivamente, all'interazione con gli accompagnatori. Il contesto più informale, inoltre, rispetto a quello di una lezione frontale, facilita la partecipazione diretta e la richiesta di spiegazioni e chiarimenti.

\section{La fabbrica e il lavoro nei documenti}

Un ulteriore blocco di attività invita gli studenti a riflettere sui documenti che ci aiutano a ricostruire la storia del lavoro e della comunicazione industriale. In particolare, alla classe sono proposte due diverse modalità di conservazione e valorizzazione del patrimonio documentario: un archivio e un museo aziendale.

Presso la Fondazione gli studenti sperimentano un laboratorio sulle fonti: gli archivisti presentano i principali fondi industriali conservati in Isec, in particolare il fondo Breda, e illustrano i principali strumenti e compiti del lavoro archivistico. Poi gli studenti sono invitati ad analizzare autonomamente, in piccoli gruppi, seguendo una traccia proposta, le copie di alcuni documenti conservati in istituto, traendone il maggior numero di informazioni possibili.

Presso il Museo Campari, invece, gli studenti scoprono la realtà di un'azienda alimentare, l'unica sopravvissuta tra le grandi fabbriche sestesi, e una modalità completamente diversa di narrarne la storia attraverso i documenti. Una visita guidata li accompagna all'interno del museo in cui sono esposti non solo i materiali della comunicazione (quadri, manifesti, pannelli pubblicitari) ma anche gli oggetti prodotti. L'azienda non si offre più allo sguardo attraverso ciò che si è salvato senza alcuna precisa intenzione e volontà (carte, fotografie, parti di macchinari, ruderi di capannoni...) ma seleziona e decide le modalità della propria autorappresentazione. Il lavoro scompare, ne rimane l'eco patinata e attraente del consumo. 


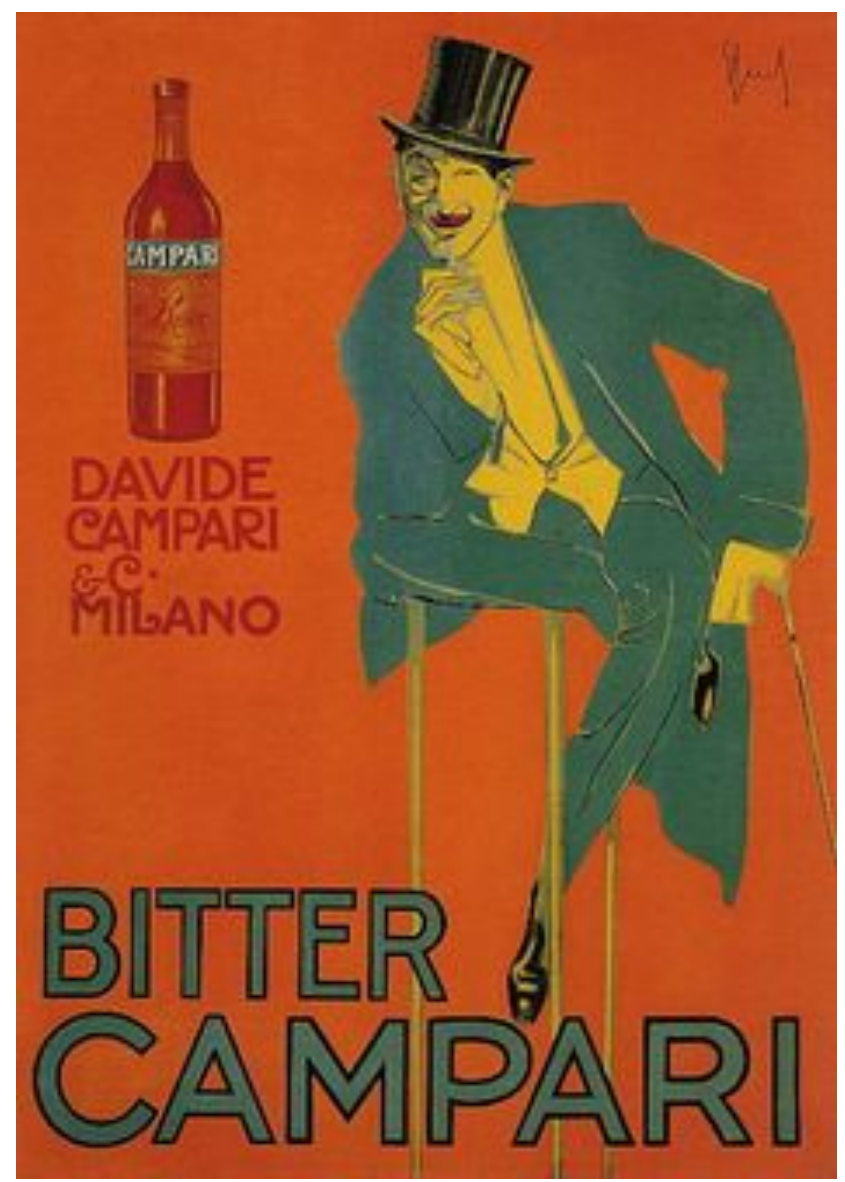

Un manifesto pubblicitario della Campari (F. Laskoff, 1904)

I testimoni: gesti e pensieri del lavoro

Raccontare le difficoltà, la fatica, le tensioni, raccontare il lavoro, non è semplice. Per questo a spazi e documenti, abbiamo deciso di affiancare la voce di due testimoni d'eccezione: Uliano Lucas e Canio Di Ruggiero.

Lucas non è stato solo uno tra i più importanti fotoreporter italiani ma anche uno tra coloro che più si sono spesi nella rappresentazione del lavoro e più hanno fotografato all'interno degli spazi produttivi. La sua testimonianza, accompagnata dalla proiezione di una selezione delle sue immagini, ha, dunque, una doppia valenza. Da una parte è il racconto delle modalità e dei cambiamenti che hanno investito il lavoro dall'inizio degli anni Settanta fino al nuovo millennio, oltre che delle tensioni e dei conflitti sociali che di questo mondo sono stati espressione. Dall'altro, è il racconto di un lavoro, quello del fotogiornalista, anch'esso al centro di cambiamenti radicali negli ultimi anni, e del quale gli studenti spesso non hanno alcuna conoscenza.

Il secondo testimone che i ragazzi hanno incontrato, Di Ruggiero, ha raccontato la propria esperienza: prima immigrato dalla Puglia, confuso tra le migliaia di operai che hanno popolato Sesto San Giovanni nel secondo dopoguerra, poi sindacalista alla Falck, impegnato dapprima a rappresentare necessità e rivendicazioni operaie durante gli anni della grande produzione e del boom economico e poi, al culmine della crisi, nella complessa e delicata fase della chiusura degli stabilimenti e della dismissione. 
Saper leggere un luogo. I segni della memoria collettiva e l'identità del presente. Mostra sull'area archeologica industriale ex Breda attraverso la documentazione storica di Fondazione Isec è il titolo completo del secondo caso proposto. Si tratta di un progetto di Alternanza scuola lavoro svolto in collaborazione tra Fondazione Isec e il Liceo artistico statale di Brera.

La classe partecipante, dell'indirizzo architettura e ambiente, ha lavorato per due anni, e un totale di 45 ore sulle aree dismesse della Breda, analizzandole e progettandone poi riutilizzi e riallestimenti urbani, concludendo il percorso nell'anno scolastico 2018-2019.

Il percorso si è aperto con una serie di incontri introduttivi sulla fabbrica e il lavoro nel Novecento, con particolare riferimento al passaggio al post-fordismo e, infine, alla deindustrializzazione. Un ulteriore incontro è servito a presentare la particolare vicenda di Sesto e, al suo interno, della storia della Società italiana Ernesto Breda per costruzioni meccaniche, poi, dagli anni Cinquanta, Finanziaria Breda Spa.

In seguito, accompagnati dalla docente di classe e da alcuni esperti, i ragazzi si sono recati sulle aree dismesse per una prima ispezione, seguita da una successiva serie di sopralluoghi utili a compiere i rilievi delle aree interessate dal progetto. Fra queste, lo Spazio Museo dell'Industria e del lavoro, adiacente al Carroponte e all'area di archeologia industriale dell'ex Breda.[3] Con l'aiuto del personale di Fondazione Isec, hanno poi imparato a conoscere l'archivio dell'istituto e hanno scoperto come usarlo. Sempre accompagnati dagli archivisti, hanno poi effettuato una ricerca sul materiale archivistico che avrebbe potuto fungere da supporto al loro percorso. All'osservazione diretta si sono quindi affiancate le fonti storiche.

\section{Restituire la città alla città}

Tutto il materiale raccolto in questa prima parte del progetto è stato utilizzato per documentare la situazione in essere e ipotizzare possibili soluzioni alternative per il recupero degli spazi. La tappa conclusiva ha visto, poi, gli studenti mettersi alla prova con un piccolo ma concreto progetto: l'allestimento di una mostra negli spazi di Fondazione Isec. Disegni, fotografie, progetti sono stati combinati in una serie di pannelli che sono rimasti a disposizione del pubblico per alcune settimane. 


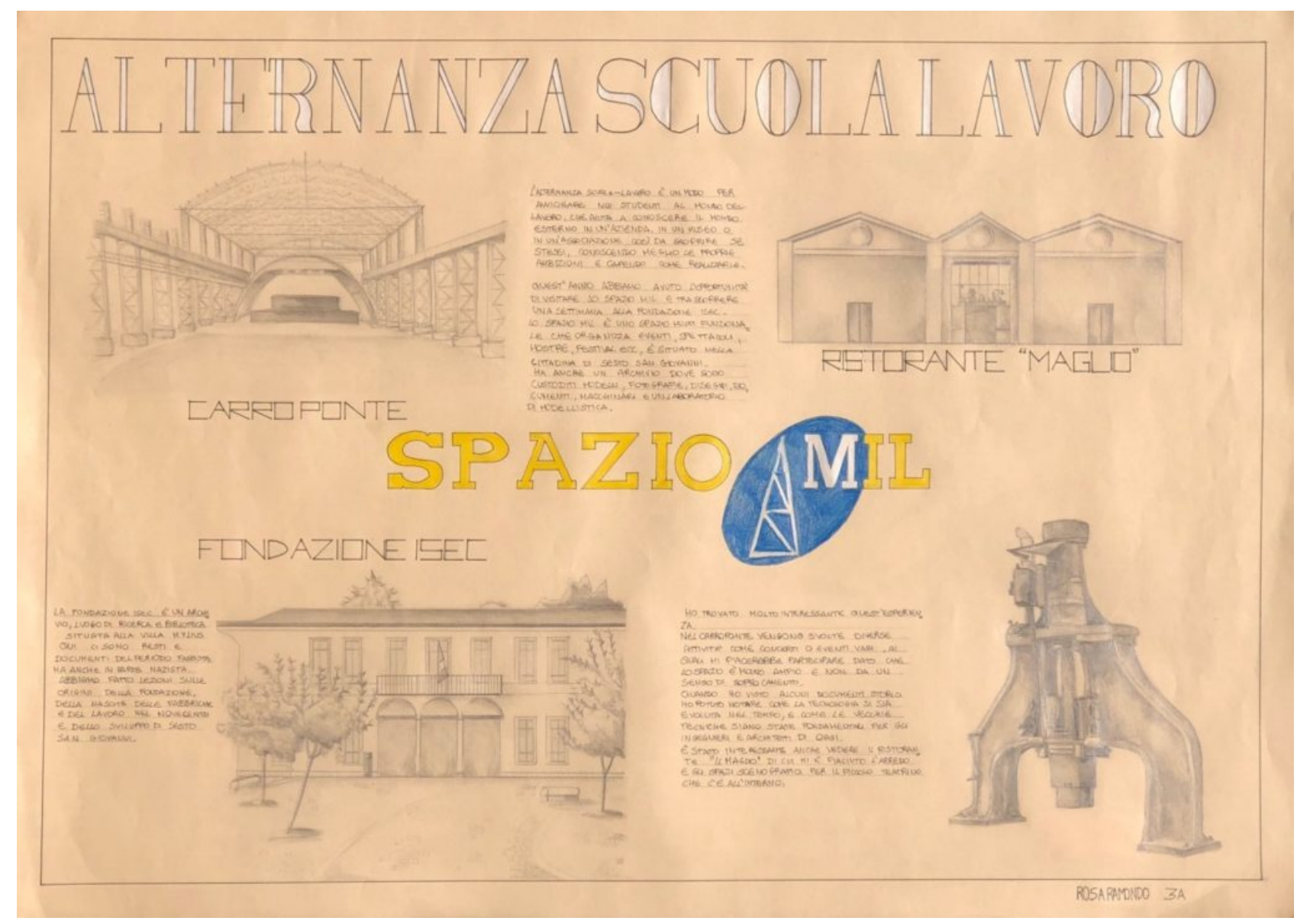

Un pannello della mostra conclusiva (Rosa Ramondo, 2019)

Il percorso ha ottenuto così due obiettivi: da una parte ha offerto alla classe la possibilità di mettersi alla prova su un progetto concreto, creando un prodotto che documentasse l'acquisizione di alcune specifiche competenze, sia strettamente legate al profilo professionale che di ambito più allargato, e che fosse condivisibile. Con i compagni, i docenti, gli amici, i familiari ma anche con la città sul cui territorio avevano lavorato.

E questo è il secondo obiettivo. La prova finale ha consentito alla città di ri-guardare, attraverso gli occhi degli studenti e delle studentesse, una parte della propria storia e di ipotizzare percorsi alternativi in quegli spazi reali, abbandonati o attraversati nella propria vita quotidiana. Non si è trattato, dunque, solamente, di un modo per consentire ai discenti di attivare in un compito di realtà le proprie abilità e competenze ma anche un modo per la Fondazione di restituire alla città una parte di quei documenti e di quella storia che la città stessa ha prodotto. 


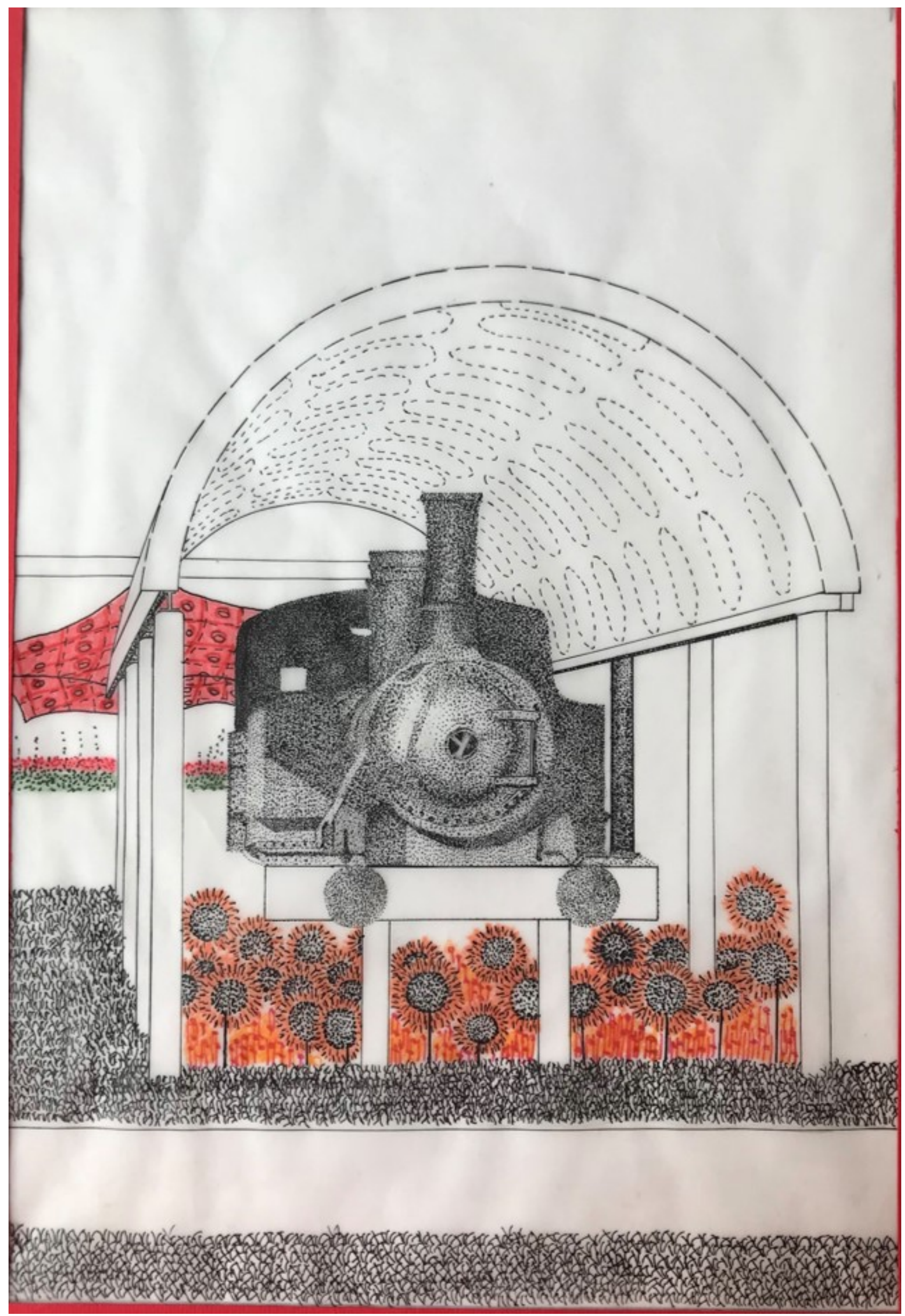




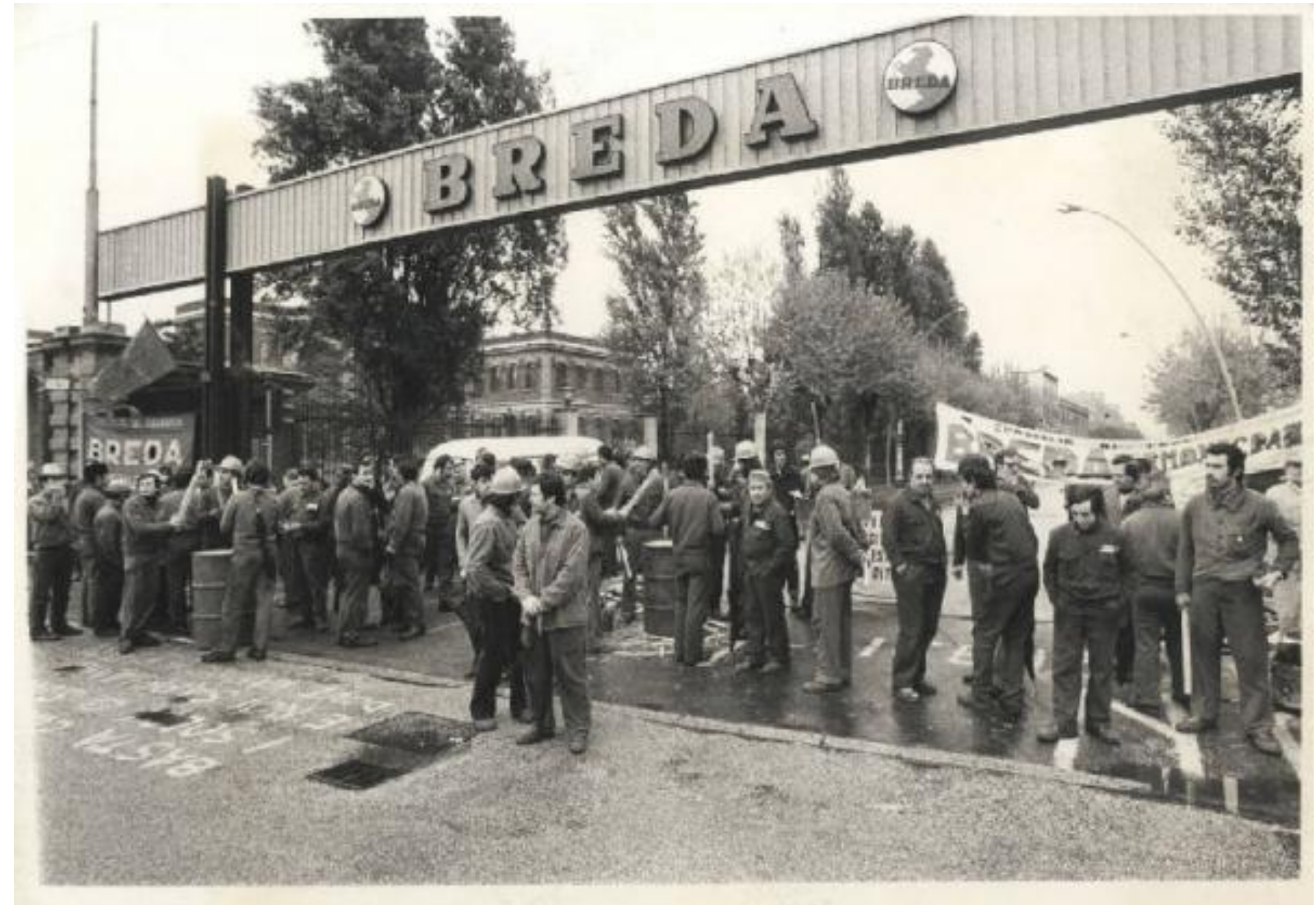

Breda termomeccanica e locomotive, presidio dei lavoratori, Sesto San Giovanni, 29 aprile 1976, (Fondazione ISEC, Sezione fotografica dell'Archivio storico Breda, C_SC_020)

\section{Reti e saperi}

In generale, raccontare il lavoro è un'impresa complessa. Proprio per questo è necessario attivare saperi plurimi che possano offrirne visioni articolate. È anche indispensabile coinvolgere studentesse e studenti in un percorso che inviti alla partecipazione diretta e non all'ascolto passivo. Se questo vale, infatti, per qualsiasi argomento, è ancora più vero per un soggetto apparentemente ostico e lontano dagli interessi degli adolescenti.

Per rendere possibile questo processo virtuoso è necessario coinvolgere luoghi, esperienze e professionalità diverse con le quali vi siano elementi di una visione condivisa. Bisogna, quindi, avere nel tempo costruito una rete di contatti in una stretta connessione con il territorio. Del territorio e della sua storia, è inoltre necessario avere costruito nel tempo, con il lavoro sui documenti e la ricerca, una conoscenza approfondita che consenta di leggerne e trasmetterne la traccia anche a persone lontane, generazionalmente e per formazione, dalle tematiche affrontate.

\section{Note:}

[1] Cfr. Percorsi per le competenze trasversali e per l'orientamento. Linee guida, MiurDipartimento per il sistema educativo di istruzione e formazione, 4 settembre 2019, 
https://www.miur.gov.it/documents/20182/1306025/Linee+guida+PCTO+con+allegati.pdf/3e6b551 4-c5e4-71de-8103-30250f17134a?version $=1.0 \& \mathrm{t}=1570548388496$, url consultata il 15 febbraio 2020.

[2] Legge 92 del 20 agosto 2019, in "Gazzetta ufficiale della Repubblica italiana. Serie generale", 195, 2019, https://www.gazzettaufficiale.it/eli/id/2019/08/21/19G00105/sg, url consultata il 15 febbraio 2020.

[3] Si veda Spazio Mil, http://www.spaziomil.org/?doing_wp_cron=1583769317.1451609134674072265625, url consultata il 15 febbraio 2020. 